PUBLICATIONS

\section{FROM THE BENCH TO THE BOARDROOM}

Scientist as Eatrepreneurs. Rarel J. Samsom. ISBN 0-79239111-X. \$45 (Kluwer Academic Publishers, Boston: 1990)

$\mathrm{W}$ hy do scientists want to become involved in commercializing their ideas? How do they go about it? Do they retain their relationships with academic institutions? What determines whether they succeed or fail? These are some of the key questions posed by Karel Samsom in his survey of 22 scientist-started ventures in the states of Massachusetts, Maine, New Hampshire, Vermont, and the Canadian provinces of Ontario and Quebec. Among those who participated in the study were Henry E. Blair (Genzyme), Walter Gilbert (Biogen), Thomas G. Adelman (ImmuneCell), and David S. Dime (Toronto Research Chemicals).

One of the key findings is that scientists and entrepreneurial managers have different perceptions of time and planning; they also have different value systems concerning money, management, technology development, and commercialization. Thus, scientists have perceptions of organizational goals very different from managers. Scientists tend to work for a "greater cause" than just company objectives, concentrating on the technology per se rather than the running of a business. Managers, on the other hand, tend to view the technology as one of the many ingredients in the commercialization process. But scientists and entrepreneurs do share some traits: they are willing to risk their own resources to pursue commercial opportunities, and they want monetary rewards.

The survey also showed that ventures focused entirely on technology often fumbled when it came to team integration, finance, administration, and marketing. The allocation of insufficient staff and resources to "business" concerns tends to be "a serious trap," sometimes causing the business to fail. And although most of the scientist-entrepreneurs admitted-either implicitly or explicitly-that they really should learn something about business, especially in the areas of team management and finance, they failed to act on this knowledge.

Also, profound differences in value systems between scientists and financiers kept popping up. And these differences create tension for the scientist, torn between the requirements of the financial partner and the need to independently pursue science.

The survey also determined that most of the scientists wanted to maintain good relationships with their universities. In practice this only occurred if the researcher retained a full-time position and worked in the entrepreneurial venture during "spare time."

Overall, the book offers some valuable insights and tips for any scientist thinking about starting his or her own company. Ten years ago, it would have been even better, since-at least in biotechnology-there have been enough startups for the lessons to have become entered the professional lore.
Where to find Gilson

high performance pipetting systems

\begin{tabular}{|c|c|}
\hline Australia & $\begin{array}{l}\text { JOHN MORRIS PTY LTD } \\
\text { P.O. Box 447, WILLOUGHBY NSW } 2068 \\
\text { Tel. : } 61 \text { (2) } 4176466 \text { - Fax : } 61 \text { (2) } 4178855\end{array}$ \\
\hline Austria & $\begin{array}{l}\text { P. HAACK LABORBEDARF } \\
\text { Garnisongasse } 3,1096 \text { VIENNA } \\
\text { Tel. : } 43 \text { (222) } 434606 \text { - Fax : } 43 \text { (222) } 42120128\end{array}$ \\
\hline Belgium & $\begin{array}{l}\text { ANALIS } \\
\text { Leeuwerikstraat } 28 \text { - } 9000 \text { GENT } \\
\text { Tel. : } 32 \text { (91) } 225866 \text { - Fax : } 32 \text { (91) } 200848\end{array}$ \\
\hline Canada & $\begin{array}{l}\text { MANDEL SCIENTIFIC COMPANY LTD. } \\
\text { Hanlon Business Park, } 2 \text { Admiral Place, R.R. \# } 6 \\
\text { GUELPH - ONTARIO N1H 6J3 } \\
\text { Tel. : } 1 \text { (519) 763-2145 - Fax : } 1 \text { (519) 763-2005 }\end{array}$ \\
\hline $\begin{array}{l}\text { China } \\
\text { (People's } \\
\text { Republic of) }\end{array}$ & $\begin{array}{l}\text { WORLD WAYS Co. LTD. } \\
\text { 172-176 Wing Lok Street } \\
\text { Winfull Commercial Bldg-2nd Floor, HONG KONG } \\
\text { Tel. : } 852 \text { (5) } 430760 \text { - Fax : } 852 \text { (5) } 5414330\end{array}$ \\
\hline Denmark & $\begin{array}{l}\text { BIOLAB APS } \\
\text { Sindalsvej 30, DK } 8240 \text { RISSKOV } \\
\text { Tel. : } 45 \text { (86) } 212866 \text { - Fax : } 45 \text { (86) } 212301\end{array}$ \\
\hline $\begin{array}{l}\text { Eastern } \\
\text { Europe }\end{array}$ & $\begin{array}{l}\text { CHEMINST } \\
\text { Laudongasse } 40,1080 \text { VIENNA, AUSTRIA } \\
\text { Tel. : } 43 \text { (222) } 434483 \text { - Fax : } 43 \text { (222) } 43448326\end{array}$ \\
\hline France & $\begin{array}{l}\text { GILSON MEDICAL ELECTRONICS (France) S.A. } \\
\text { B.P. } 45,95400 \text { VILLIERS-LE-BEL } \\
\text { Tel. : } 33 \text { (1) } 34295050 \text { - Fax: } 33 \text { (1) } 34295060\end{array}$ \\
\hline Germany & $\begin{array}{l}\text { ABIMED ANALYSEN TECHNIK GmbH } \\
\text { Raiffeisenstrasse } 3 \text {, Postfach } 2141,4018 \text { LANGENFELD } \\
\text { Tel. : } 49 \text { (2173) } 89050 \text { - Fax : } 49 \text { (2173) } 890577\end{array}$ \\
\hline India & $\begin{array}{l}\text { A.S.R. INSTRUMENTS } \\
28 \text { Sankey Road, BANGALORE } 560052 \\
\text { Tel. : } 91 \text { (812) } 267931 \text { - Fax : } 91 \text { (812) } 267676\end{array}$ \\
\hline Israel & $\begin{array}{l}\text { LANDSEAS LTD } \\
\text { P.O. Box } 23011 \text { - } 38 \text { King George Street, TEL AVIV } 612 \\
\text { Tel. : } 972 \text { (3) } 299091 \text { - Fax : } 972 \text { (3) } 282651\end{array}$ \\
\hline Italy & $\begin{array}{l}\text { BIOLABO INSTRUMENTS SRL } \\
\text { Via Braccio da Montone } N^{\circ} 6,20154 \text { MILANO } \\
\text { Tel. : } 39 \text { (2) } 3493251 \text { - Fax } 39 \text { (2) } 347900\end{array}$ \\
\hline Japan & $\begin{array}{l}\text { M \& S INSTRUMENTS TRADING INC. } \\
\text { 12-4 Mikuni-Honmachi, 2-Chome, } \\
\text { Yodogawa-Ku, OSAKA 532 } \\
\text { Tel. : } 81 \text { (6) 396-0501 - Fax : } 81 \text { (6) } 3952588\end{array}$ \\
\hline Netherlands & $\begin{array}{l}\text { MEYVIS EN Co. B.V. } \\
\text { Postbus } 265 \text { - Pergosilaan } 4 \\
4600 \text { AG BERGEN OP ZOOM } \\
\text { Tel. : } 31 \text { (1640) } 36922 \text { - Fax : } 31 \text { (1640) } 66651\end{array}$ \\
\hline Portugal & $\begin{array}{l}\text { REAGENTE V } \\
\text { Rua Damiao de Gois No } 358 \text {, Apartado } 4710 \\
4012 \text { PORTO CODEX } \\
\text { Tel. : } 351 \text { (2) } 486185 \text { - Telex : } 28167\end{array}$ \\
\hline South Africa & $\begin{array}{l}\text { LABORATORY \& SCIENTIFIC EQUIPMENT Co. PTY LT } \\
\text { P.O. Box } 2110 \text {, CAPE TOWN } 8000 \\
\text { Tel. : } 27 \text { (21) } 5317504 \text { - Fax : } 27 \text { (21) } 5317562\end{array}$ \\
\hline Spain & $\begin{array}{l}\text { PACISA } \\
\text { Calle Ayala 83, MADRID } 28006 \\
\text { Tel. : } 34 \text { (1) } 4027060 \text { - Fax : } 34 \text { (1) } 4028938\end{array}$ \\
\hline Sweden & $\begin{array}{l}\text { INSTRUMENT AB LAMBDA } \\
\text { Box 609, } 19126 \text { SOLLENTUNA } \\
\text { Tel. : } 46 \text { (8) } 960440 \text { - Fax : } 46 \text { (8) } 356125\end{array}$ \\
\hline Switzerland & $\begin{array}{l}\text { SYNMEDIC AG } \\
\text { Seebahnstrasse } 85, \mathrm{CH}-8036 \text { ZURICH } \\
\text { Tel. : } 41 \text { (1) } 4636990 \text { - Fax : } 41 \text { (1) } 4612053\end{array}$ \\
\hline $\begin{array}{l}\text { United } \\
\text { Kingdom }\end{array}$ & $\begin{array}{l}\text { ANACHEM LTD. } \\
20 \text { Charles Street, LUTON, BEDFORDSHIRE LU2 OEB } \\
\text { Tel. : } 44 \text { (582) } 456666 \text { - Fax : } 44 \text { (582) } 391768\end{array}$ \\
\hline U.S.A. & $\begin{array}{l}\text { RAININ INSTRUMENT Co. Inc. } \\
\text { Mack Road, WOBURN, MA } 01801 \\
\text { Tel. : } 1 \text { (617) 935-3050 - Fax : } 1 \text { (617) 938-8157 }\end{array}$ \\
\hline U.S.S.R. & $\begin{array}{l}\text { CHEMINST } \\
\text { Sovincenter Appt. } 737 \text {, Mej. No 2, } 123610 \text { MOSCOW } \\
\text { Tel. : } 70 \text { (95) } 2532737 \text { - Telex : } 411432\end{array}$ \\
\hline
\end{tabular}

If your country is not listed, please contact : GILSON MEDICAL ELECTRONICS (France) S.A. 72, rue Gambetta, BP 45, 95400 Villiers-le-Bel, France Tel. : 33 (1) 34295000 - Fax : 33 (1) 34295080 Circle No. 139 on Reader Service Card 